\title{
Contributed Papers for JD15: Abstracts
}

\section{The Evolution of the $\mathrm{C} / \mathrm{O}$ ratio in Metal-Poor Halo Stars}

C.J. Akerman ${ }^{1}$, L. Carigi ${ }^{2}$, P.E. Nissen ${ }^{3}$, M. Pettini ${ }^{1}$, M. Asplund ${ }^{4}{ }^{1}$ Institute of Astronomy, Cambridge, UK; ${ }^{2}$ Instituto de Astronomía, UNAM, México;

${ }^{3}$ University of Aarhus, Denmark; ${ }^{4} \mathrm{MSSSO}$, Australia.

Abstract. We report new measurements of carbon and oxygen abundances in 34 dwarf and subgiant Galactic halo stars with metallicities $[\mathrm{Fe} / \mathrm{H}]=-0.7$ to -3.2 . We observed four permitted lines of $\mathrm{C}$ I near $9100 \AA$ and the O I $7774 \AA$ triplet, all recorded at high signal-to-noise ratios with the UVES echelle spectrograph on the ESO VLT. The line equivalent widths were analyzed with the 1D, LTE, MARCS model atmosphere code to deduce $\mathrm{C}$ and $\mathrm{O}$ abundances; corrections due to non-LTE and 3D effects are discussed. Our survey has uncovered tentative evidence to suggest that, as the oxygen abundance decreases below $[\mathrm{O} / \mathrm{H}]=$ $-1,[\mathrm{C} / \mathrm{O}]$ may not remain constant at $[\mathrm{C} / \mathrm{O}]=-0.5$, as previously thought, but increase again, possibly approaching near-solar values at the lowest metallicities. With the current dataset this is no more than a 3 sigma effect and it may be due to metallicity-dependent non-LTE corrections to the $[\mathrm{C} / \mathrm{O}]$ ratio which have not been taken into account. However, its potential importance as a window on the nucleosynthesis by Population III stars is a strong incentive for future work, both observational and theoretical, to verify its reality. (see Akerman et al. 2003, A\&A, 414, 931; astro-ph/0310472)

\section{Subaru HDS Studies of Carbon-Rich, Very Metal-Poor Stars}

W. Aoki ${ }^{1}$, S.G. Ryan ${ }^{2}$, J.E. Norris ${ }^{3}$, T. Beers ${ }^{4}$, S. Tsangarides ${ }^{2}$, H. Ando ${ }^{1}$ ${ }^{1}$ National Astronomical Observatory of Japan, Tokyo; ${ }^{2}$ The Open University, UK; ${ }^{3} \mathrm{MSSSO}$, Australia; ${ }^{4}$ Michigan State University, USA.

Abstract. Recent surveys of metal-poor stars have discovered a large number of carbon-rich objects. To investigate the origin of the carbon excesses, detailed abundance studies were made for 33 carbon-rich, metal-poor stars based on high-resolution spectra obtained with the Subaru Telescope (a part of the results was reported by Aoki et al. 2002, ApJ, 580, 1149) and the Anglo-Australian Telescope (Aoki et al. 2002, ApJ, 567, 1166). 24 stars in our sample (approximately $70 \%$ ) show large excesses of $s$-process elements (e.g., Ba). A correlation between $\mathrm{C}$ and $\mathrm{Ba}$ abundances in these objects suggests production of carbon and $s$-process elements in the same site, probably in thermally pulsing AGB stars, and the dilution of the yields. The other 9 objects have low (or normal) $\mathrm{Ba}$ abundance. One of them (CS29498-043) has extremely low iron abundance $([\mathrm{Fe} / \mathrm{H}]=-3.7)$ and significant over-abundances of $\mathrm{Mg}$ and $\mathrm{Si}$ (Aoki et al. 2002, ApJ, 576, L141). This chemical nature is suggestive of supernovae production in which relatively little material escaped from the region surrounding the iron core. The other 8 carbon-rich, Ba-normal stars simply show an excess of $\mathrm{C}$ (or excesses of $\mathrm{C}$ and $\mathrm{N}$ ). Some models (e.g., supernovae with extreme mixing in the explosion, He-flash in low-mass red-giants) are proposed to explain the carbon-enhancement in these stars. 


\section{Rapid Neutron Capture Process in Supernovae}

R. Baruah ${ }^{1}$, K. Duorah ${ }^{2}$, H.L. Duorah ${ }^{2}{ }^{1}$ Department of Physics, HRH Prince of Wales Inst. of Engg. and Technology, India; ${ }^{2}$ Department of Physics, Gauhati University, India.

Abstract. The rapid neutron capture process is one of the major nucleosynthesis processes responsible for the synthesis of heavy nuclei beyond iron. Approximately half of the heavy elements with mass number $A>70$ and all of the actinides in the solar system are believed to have been produced in the $r$ process. We have studied the $r$-process in supernovae for production of heavy elements beyond $A=45$. The supernova envelopes at temperatures $T>10^{9} \mathrm{~K}$ and neutron density $n \sim 10^{24} \mathrm{~cm}^{-3}$ are considered to be one of the most potential site for the $r$-process. The primary goal of the calculations is to fit the global abundance curve for solar system $r$-process isotopes by varying time dependent parameters such as temperature and neutron density. The neutron capture paths are obtained at different $Q_{n}$ values ranging from $0.52 \mathrm{MeV}$ to 5.07 $\mathrm{MeV}$. We have studied the abundance distributions corresponding to temperatures ranging from $1.2 \times 10^{9} \mathrm{~K}$ to $2.8 \times 10^{9} \mathrm{~K}$ and neutron density from $10^{24} \mathrm{~cm}^{-3}$ to $10^{32} \mathrm{~cm}^{-3}$. With temperature and density conditions of $T=2.8 \times 10^{9} \mathrm{~K}$ and $n=10^{24} \mathrm{~cm}^{-3}$ a nucleus of mass 252 was theoretically found. It was found that abundances at $Q_{n}$ from 0.52 to $4.9 \mathrm{MeV}$ give significant results. As we increase the neutron density, the computed abundance curves were found to approach the observed one in a more significant way. The beta decay of ${ }_{21} \mathrm{Sc}^{45}$ (normalization to $A=45$ ) was found to govern most of the abundance curves.

\section{The Metallicity Distribution Function of Halo Stars}

T.C. Beers ${ }^{1}$, N. Christlieb ${ }^{2}{ }^{1}$ Michigan State University, USA; ${ }^{2}$ Hamburger Sternwarte, Germany.

Abstract. Over the past two decades, a worldwide effort to obtain mediumresolution spectroscopic confirmation of candidate low-metallicity stars in the halo and thick disk of the Galaxy has produced $\sim 80001-2 \AA$ observations of stars selected from the HK objective prism survey of Beers and colleagues. More recently, the Hamburg/ESO prism survey of Christlieb and collaborators has produced a larger, and better understood, selection of metal-poor candidates that explore a much larger volume of the Galaxy than was available to the HK survey. We summarize the final derived Metallicity Distribution Function (MDF) of the HK survey objects, and compare it with that obtained from the first several years of the HES follow-up effort. In particular, we investigate whether there is evidence for a change in the nature of the MDF as a function of distance from the Galactic center, which could have profound implications for the nature of the formation and evolution of the Milky Way, and for galaxy formation in general.

\section{HERES: The Search for $r$-process Enhanced, Metal-Poor Stars} T.C.Beers ${ }^{1}$, N. Christlieb ${ }^{2}$, M.S. Bessell ${ }^{3}$, V. Hill ${ }^{4}$, P.S.Barklem ${ }^{5}$, S.G. Ryan ${ }^{6}$, S. Rossi ${ }^{7}$, A. Korn ${ }^{8}{ }^{1}$ Michigan State University, USA; ${ }^{2}$ Hamburger Sternwarte, Germany; ${ }^{3}$ MSSSO, Australia; ${ }^{4}$ Observatoire de Paris-Meudon, France; ${ }^{5}$ Uppsala Observatory, Sweden; ${ }^{6}$ The Open University, UK; ${ }^{7}$ IAG, Brazil; ${ }^{8}$ München Sternwarte, Germany. 


\begin{abstract}
In recent years, a handful of extremely metal-deficient stars have been identified that exhibit moderate to large enhancements of their abundance ratios (relative to $\mathrm{Fe}$ ) of elements associated with the astrophysical $r$-process, enabling detections of radioactive species such as $U$ and Th. Our understanding could be greatly improved by increasing the numbers of known $r$-processenhanced, metal-poor stars, as well from building the sample to the point where meaningful measures of the frequency of the phenomenon, especially as a function of metallicity, could be ascertained. We describe the present status of HERES - The Hamburg/ESO R-process Enhanced Star survey. This survey is based upon "snapshot" high-resolution VLT/UVES spectra of large numbers of giants with $[\mathrm{Fe} / \mathrm{H}]<-2.5$. Spectra of sufficient quality to detect the presence of the Eu II line (4019 $\AA$ ), a distinctive neutron-capture feature, have now been obtained for some 150-200 extremely metal-deficient giants chosen from the Hamburg/ESO survey. We discuss the number of moderate- and highly $r$-process enhanced stars discovered, update our estimate of the frequency of their detection, and present a discussion of the distribution of $\sim 20$ other easily measured elements in each of these stars (e.g., C, Ca, Mg, Si, Co, Ni, Sr, Ba, etc.).
\end{abstract}

\title{
Carbon Abundances of Metal-Poor Stars in the Galactic Halo
}

T.C. Beers ${ }^{1}$, S. Rossi ${ }^{2}$, C. Sneden ${ }^{3}$, N. Christlieb $^{4}$, J. Rhee ${ }^{5}$, S.G. Ryan ${ }^{6}$, T. Sevastianenko ${ }^{1}$, B. Marsteller ${ }^{1}{ }^{1}$ Michigan State Univ., USA; ${ }^{2}$ IAG, Brazil; ${ }^{3}$ Univ. of Texas, USA; ${ }^{4}$ Hamburger Sternwarte, Germany; ${ }^{5}$ Univ. of Virginia, USA $;{ }^{6}$ Open University, UK.

Abstract. Very metal-deficient stars that exhibit enhancements of their carbon abundances are of crucial importance for understanding a number of issues: The nature of stellar evolution among the first generations of stars, the shape of the Initial Mass Function, and the relationship between carbon enhancement and neutron-capture processes, in particular the astrophysical s-process. One fundamental result from recent objective-prism surveys dedicated to the discovery of metal-deficient stars is that the frequency, and perhaps, the level, of carbon enhancement increases greatly with declining metallicity. Most previous discoveries of these important stars have been serendipitous, as the stars were initially targeted because of their apparently low overall metallicity, and it was only discovered later that carbon was strongly enhanced. To more completely explore this phenomenon, we have undertaken spectroscopic follow-up of a published list of metal-deficient candidates from the Hamburg/ESO prism survey that show clearly strong carbon features directly on the survey plates. We have already obtained spectra for some 350 of the 413 stars in the sample, and will report on their observed properties, including estimates of their $[\mathrm{Fe} / \mathrm{H}]$ and $[\mathrm{C} / \mathrm{Fe}]$, their radial velocities, and their spatial distribution.

\section{$\mathrm{Cu}$ and $\mathrm{Zn}$ Abundances in Metal-Poor Stars}

G. Bihain ${ }^{1}, R$. Rebolo ${ }^{1}$, G. Israelian ${ }^{1}{ }^{1}$ Instituto de Astrofisica de Canarias, Spain.

Abstract. We present $\mathrm{Cu}$ and $\mathrm{Zn}$ abundances for a sample of 38 FGK stars, mostly dwarfs, spanning a metallicity range between solar and $[\mathrm{Fe} / \mathrm{H}]=-3$. LTE 
abundances were obtained using Kurucz's model atmospheres and the near-UV lines of $\mathrm{Cu}$ I $3273.95 \AA$ and $\mathrm{Zn}$ I $3302.58 \AA$ observed at high spectral resolution. The trend of $[\mathrm{Zn} / \mathrm{Fe}]$ vs. $[\mathrm{Fe} / \mathrm{H}]$ is essentially solar for $[\mathrm{Fe} / \mathrm{H}]>-2.0$ and then slightly increases at lower metallicities to an average value of $\langle[\mathrm{Zn} / \mathrm{Fe}]\rangle$ $=+0.18$, whereas the $[\mathrm{Cu} / \mathrm{Fe}]$ trend is approximately constant down to $[\mathrm{Fe} / \mathrm{H}]$ $\sim-1$ and then decreases at lower metallicities reaching a plateau around $[\mathrm{Cu} / \mathrm{Fe}]$ $\sim-0.95$ for $[\mathrm{Fe} / \mathrm{H}]<-2.5$. We compare our results with previous work on these elements and briefly discuss them in terms of nucleosynthesis processes. A paper will be submitted to A\&A.

\section{Lithium Abundances in Extremely Metal-Poor Stars}

P. Bonifacio ${ }^{1}$, P. Molaro ${ }^{1}$, T. Sivarani ${ }^{1}$, M. Spite ${ }^{2}$, F. Spite ${ }^{2}$, R. Cayrel $^{2}$, P. François ${ }^{2}$, V. Hill ${ }^{2}$, B. Plez ${ }^{3}$, T. C. Beers ${ }^{4}$, J. Andersen ${ }^{5}$, B. Barbuy ${ }^{6}$, E. Depagne ${ }^{7}$, B. Nordström ${ }^{5,8}$, F. Primas ${ }^{7}{ }^{1}$ INAF-OA Trieste; ${ }^{2}$ Observatoire de Paris-Meudon; ${ }^{3}$ GRAAL-Université de Montpellier; ${ }^{4}$ Michigan State University; ${ }^{5}$ Astronomical Observatory, Copenhagen; ${ }^{6}$ Universidade de São Paulo; ${ }^{7}$ European Southern Observatory; ${ }^{8}$ Lund Observatory, Sweden.

Abstract. We present preliminary lithium abundances for 21 turnoff stars in the range $-3.6<[\mathrm{Fe} / \mathrm{H}]<-2.5$, observed with VLT/UVES. Effective temperatures were derived by fitting the wings of the $\mathrm{H} \alpha$ lines. In 5 stars, $\mathrm{Li}$ is depleted by modest to large factors, while the remaining 16 stars define a very tight relation in the $[\mathrm{Fe} / \mathrm{H}]-\mathrm{A}(\mathrm{Li})$ plane. The sample has mean lithium abundance $\mathrm{A}(\mathrm{Li})=2.30$ with a dispersion of $0.08 \mathrm{dex}$. The relation exhibits a significant slope $(0.17 \pm 0.05)$, which cannot be simply interpreted as due to Li production in the early Galaxy: extrapolation to $[\mathrm{Fe} / \mathrm{H}]=-5.0$ implies $\mathrm{A}(\mathrm{Li})=1.94$, while the $\mathrm{A}(\mathrm{Li})$ implied by the WMAP baryonic density is $\mathrm{A}(\mathrm{Li})=2.66$ and the minimum abundance predicted by $\mathrm{BBN}$ is $\mathrm{A}(\mathrm{Li})=2.05$. More likely explanations of the steep slope are: 1) systematic errors in our analysis, 2) observational bias, or 3 ) metallicity-dependent atmospheric effects which alter the Li abundance. The data also show trends of increasing $[\mathrm{Fe} / \mathrm{H}]$ and $\mathrm{A}(\mathrm{Li})$ in the apparently brighter stars, which could be partly due to observational bias. Accordingly, we defer a full discussion of the slope of the $[\mathrm{Fe} / \mathrm{H}]-\mathrm{A}(\mathrm{Li})$ relation until these effects are fully understood.

\section{Spin Temperature in High Redshift DLAs}

J. Chengalur ${ }^{1}, N$. Kanekar ${ }^{2}$ National Center for Radio Astrophysics, Pune University, India; ${ }^{2}$ Kapteyn Institute, University of Groningen, The Netherlands.

Abstract. We present results from recent deep searches for $\mathrm{H} \mathrm{I} 21 \mathrm{~cm}$ absorption in high redshift Damped Lyman-alpha systems (DLAs). $21 \mathrm{~cm}$ observations, coupled with measurements of the total H I column density from the Lymanalpha line, allow one to estimate the average spin temperature $T_{s}$ of the neutral hydrogen in the DLA. In most astrophysical circumstances, the spin temperature is the same as the kinetic temperature, for a single homogeneous $\mathrm{HI}$ cloud. In a heterogeneous medium, the average spin temperature allows one to estimate the fractional $\mathrm{HI}$ content in different temperature phases (i.e. WNM and CNM). We discuss the variation in the average spin temperature with redshift, as well as morphology of the optical counterpart. Dwarf galaxies are known to contain a 
larger fraction of their atomic gas in the WNM phase, so variations of the average spin temperature with morphological type of the optical counterpart could be expected. Finally, we present preliminary results of a new survey which attempts to relate the spin temperature of an absorber to its metallicity, i.e. its $[\mathrm{Zn} / \mathrm{H}]$ ratio. (See Kanekar \& Chengalur 2003, A\&A, 399, 857 for more details.)

\section{$\mathrm{D} / \mathrm{H}$ in a New Lyman Limit Absorber towards Q1937-1009}

N.H. Crighton ${ }^{1}, J$. Web $b^{1}{ }^{1}$ Department of Astrophysics, University of New South Wales, Australia.

Abstract. Initial results from the WMAP satellite have constrained the cosmological baryon density with an uncertainty of $4 \%$. We can now test our understanding of both Big Bang nucleosynthesis and subsequent stellar nucleosynthesis by comparing this precise result with predictions for the baryon density from abundances of light elements produced in BBN. The discrepancy between the predicted baryon density from $\mathrm{He}^{4}$ and $\mathrm{Li}^{7}$ measurements and the density predicted from deuterium and WMAP already shows that there are problems either with the interpretation of the data or the model we are fitting to it. Currently the handful of extra-galactic deuterium abundance $(\mathrm{D} / \mathrm{H})$ measurements broadly agree with the WMAP baryon density, but there is significant scatter amongst these values. While the scatter can be explained by underestimated systematic effects, it may also be hinting at an early source of nucleosynthesis or other non-standard scenarios. We present a measurement of $\mathrm{D} / \mathrm{H}$ in a new Lyman limit absorption system towards QSO 1937-1009. Several different models of the velocity structure of the system require $\mathrm{D} / \mathrm{H}$ to be significantly lower than that measured in other absorption systems. If true, this may imply that the scatter in $\mathrm{D} / \mathrm{H}$ measurements is real, and not only a result of systematic errors.

\section{Fluorine in the Galaxy and the Large Magellanic Cloud}

K. Cunha ${ }^{1}$, V. Smith ${ }^{2}, K$. Hinkle ${ }^{3}{ }^{1}$ Observatorio Nacional - MCT, Brazil; ${ }^{2}$ Univ. of Texas, El Paso, USA; ${ }^{3}$ NOAO, Tucson, USA.

Abstract. The behavior of fluorine with metallicity has not yet been probed in any stellar population. To date, fluorine abundances have only been measured in a few $\mathrm{K}$ and $\mathrm{M}$ giant stars with near-solar metallicities in our Galaxy. The origins of fluorine are still uncertain and could be due to perhaps three sources: 1) neutrino-induced nucleosynthesis happening in core-collapse supernovae, 2) asymptotic giant branch stars undergoing He-burning thermal pulses, or 3) massloss from He burning regions in Wolf-Rayet stars. In this work we present first results for fluorine abundances in a sample of red giants from the Galactic disk, M4, Omega Centauri, and the LMC from spectra obtained with Phoenix on Gemini South. The observed IR spectra contain HF molecular lines. If fluorine is produced with Wolf-Rayet stars, its yield is predicted to be a strong positive function of metallicity. In such a case, fluorine abundances derived in DLA systems could test for chemical evolution driven by Wolf-Rayet stars.

\section{Probing the DLA Nature and Star Formation History} M. Dessauges-Zavadsky ${ }^{1,4}, F$. Calura ${ }^{2}, J . X$. Prochaska $^{3}$, S. D'Odorico ${ }^{4}$, 
F. Matteucci ${ }^{2}{ }^{1}$ Geneva Observatory, Switzerland; ${ }^{2}$ Dept. of Astronomy, University of Trieste, Italy; ${ }^{3} \mathrm{UCO} /$ Lick observatory, USA; ${ }^{4}$ European Southern Observatory, Germany.

Abstract. By combining UVES-VLT spectra of four DLAs toward Q0100+13, Q1331+17, Q2231-00 and Q2343+12 with HIRES-Keck spectra, we covered the total optical spectral range. This large wavelength coverage allowed us to measure the column densities of 21 ions and abundances of 15 elements $-\mathrm{N}, \mathrm{O}, \mathrm{Mg}$, $\mathrm{Al}, \mathrm{Si}, \mathrm{P}, \mathrm{S}, \mathrm{Cl}, \mathrm{Ar}, \mathrm{Ti}, \mathrm{Cr}, \mathrm{Mn}, \mathrm{Fe}, \mathrm{Ni}, \mathrm{Zn}$. This comprehensive set contrasts with the majority of DLAs for which only a handful of ions and elements is observed, and is yet necessary to constrain the photoionization and dust depletion effects to derive the intrinsic abundances of DLAs. Our analysis revealed that the DLA toward Q2343+12 requires important ionization corrections. We thus had access to the complete series of intrinsic abundances in three DLAs only and we could constrain their star formation history, age and star formation rate by a detailed comparison with the chemical evolution models of Calura, Matteucci \& Vladilo $(2003$, MNRAS 340,59$)$ for spiral and irregular/starburst galaxies. Our results show that the galaxies associated with these DLAs at $z_{\mathrm{abs}}=1.7-2.5$ are either outer regions of spiral disks or starburst/irregular galaxies with ages from 0.05 to $3.5 \mathrm{Gyr}$ and with moderate star formation rates $-2.1<\log \dot{\Psi}_{*}<-1.5$ $\mathrm{M}_{\odot} \mathrm{yr}^{-1} \mathrm{kpc}^{-2}$ (see Dessauges-Zavadsky et al. 2003, A\&A, submitted).

\section{Damped Systems Associated with a Galaxy Cluster at $\mathbf{z}=\mathbf{2 . 3 8}$}

P.J. Francis ${ }^{1}$, G. Williger ${ }^{2}{ }^{1}$ The Australian National University; ${ }^{2}$ John Hopkins Univ., USA.

Abstract. We have found a unique cluster of three damped Lyman-alpha systems at redshift 2.38, seen in absorption against three background QSOs. One of the damped systems lies in the center of a rich galaxy cluster - indeed it lies less than $150 \mathrm{kpc}$ from a pair of giant elliptical galaxies. Despite this dense environment, the gas is cold and has a metallicity of only -2.7 dex. Another damped system lies roughly 10 co-moving $\mathrm{Mpc}$ away in the middle of a void. Despite this low density location, it has a much higher metallicity: around -1.5 dex. We conclude that there is no strong correlation between the environment of damped systems and their metallicities at high redshifts. Damped systems in the middle of clusters can have very low metallicities, while damped systems in voids can be substantially enriched.

\section{Heavy Elements in a Sample of Extremely Metal-Poor Giants}

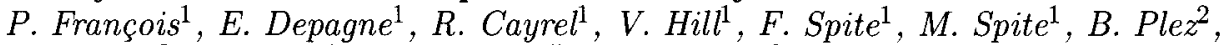
B. Barbuy ${ }^{3}$, T. Beers ${ }^{4}$, P. Bonifacio ${ }^{5}$, J. Andersen ${ }^{6}$, F. Primas ${ }^{7}$, S. Thirupath ${ }^{1}$, B. Nordströ ${ }^{6,8}$, P. Molaro ${ }^{5}{ }^{1}$ Observatoire de Paris-Meudon; ${ }^{2} \mathrm{GRAAL}$ Université de Montpellier; ${ }^{3}$ Universidade de São Paulo; ${ }^{4}$ Michigan State University; ${ }^{5}$ INAF-OA Trieste; ${ }^{6}$ Astronomical Observatory, Copenhagen; ${ }^{7}$ European Southern Observatory; ${ }^{8}$ Lund Observatory, Sweden.

Abstract. The abundances of the neutron capture elements ( $\mathrm{Sr}, \mathrm{Ba}, \mathrm{La}, \mathrm{Ce}$, $\mathrm{Eu}$...) are studied in a sample of more than 30 extremely metal-poor giants $([\mathrm{Fe} / \mathrm{H}]<-2.7)$ observed at the VLT with the high resolution spectrograph 
UVES. The $\mathrm{S} / \mathrm{N}$ ratio of the spectra is high and it is generally possible to measure very weak lines $(W>1 \mathrm{~m} \AA)$. The trends of the ratios $[\mathrm{Sr} / \mathrm{Fe}],[\mathrm{Ba} / \mathrm{Fe}]$ .. with metallicity are shown and the scatters compared to the scatter observed for iron-peak elements. Consequences for the formation of these elements and the Galactic evolution are discussed.

\section{Constraints on Early Galactic Enrichment from a Large Sample of Extremely Metal-Poor Stars Observed with VLT+UVES}

V. Hill ${ }^{1}$, R. Cayrel ${ }^{1}$, M. Spite ${ }^{1}$, B. Plez ${ }^{2}$, F. Spite ${ }^{1}$, P. François ${ }^{1}$, E. Depagne ${ }^{3}$, J. Andersen ${ }^{4}$, B. Barbuy ${ }^{5}$, T. Beers ${ }^{6}$, P. Bonifacio ${ }^{7}$, P. Molaro ${ }^{7}$, B. Nordström ${ }^{4,8}, F$. Primas ${ }^{3}{ }^{1}$ Observatoire de Paris-Meudon; ${ }^{2}$ GRAAL-Université de Montpellier; ${ }^{3}$ European Southern Observatory; ${ }^{4}$ Astronomical Observatory, Copenhagen; ${ }^{5}$ Universidade de São Paulo; ${ }^{6}$ Michigan State University; ${ }^{7}$ INAFOA Trieste; ${ }^{8}$ Lund Observatory, Sweden.

Abstract. The results from an large effort conducted at ESO-VLT+UVES to measure abundances in a sample of extremely metal-poor stars (EMPS) from high-resolution and high signal-to-noise spectra are presented. More than 70 EMPS with $[\mathrm{Fe} / \mathrm{H}]<-2.7$ were observed, equally distributed between turnoff and giants stars, and very precise abundance ratios could be derived thanks to the high quality of the data. Among the results, the abundances of the elements from $\mathrm{C}$ to $\mathrm{Zn}$ of the 35 extremely metal-poor giants of the sample (Cayrel et al., 2004, A\&A, 416, 1117), are presented, including the much debated abundance of oxygen in the early galaxy (we present [OI] line measurements down to $[\mathrm{O} / \mathrm{Fe}]=-3.5$ ), and the trends of alpha elements, iron group elements and zinc. The remarkably small scatter around these trends is also discussed, together with its implications on the early Galactic enrichment. More specific topics covered by this large effort (and large team) are addressed in devoted posters presented at this JD: Bonifacio et al., François et al., Spite et al.

\section{Molecules in Damped Ly $\alpha$ Systems: Spatial Distribution}

H. Hirashita ${ }^{1}$, A. Ferrara ${ }^{2}, K$. Wada ${ }^{3}$, P. Richter ${ }^{4}{ }^{1}$ Graduate School of Science, Nagoya University, Japan; ${ }^{2}$ SISSA, Trieste, Italy; ${ }^{3}$ National Astronomical Observatory of Japan, Tokyo; ${ }^{4}$ Osservatorio Astrofisico di Arcetri, Firenze, Italy.

Abstract. To interpret $\mathrm{H}_{2}$ (hydrogen molecule) quasar absorption line observations in damped Ly-alpha clouds (DLAs), we model the $\mathrm{H}_{2}$ spatial distribution within a DLA. Based on numerical simulations of disk structures with parameters similar to those derived for such absorbers, we calculate the $\mathrm{H}_{2}$ distribution as a function of ultraviolet background (UVB) intensity and dust-to-gas ratio. For typical values of these two quantities we find that the area in which the $\mathrm{H}_{2}$ fraction exceeds $10^{-6}$ (typical observational detection limit) only covers $<10 \%$ of the disk surface, i.e., $\mathrm{H}_{2}$ has a very inhomogeneous, clumpy distribution even at these low abundance levels. This explains the relative paucity of $\mathrm{H}_{2}$ detections in DLAs. We also show the dependence of the covering fraction of $\mathrm{H}_{2}$ on dust-to-gas ratio and UVB intensity and we comment on the physics governing the $\mathrm{H}_{2}$ chemical network at high redshift. We finally comment on our implication on the statistics of the $\mathrm{H}_{2}$ column density distribution. (See Hirashita, H., Ferrara, A., Wada, K., \& Richter, P. 2003, MNRAS, 341, L18) 


\section{Subaru/HDS Studies of $r$-process Elements in Metal-Poor Stars} S. Honda ${ }^{1}$, W. Aoki $i^{1}$, T. Kajino ${ }^{1}$, H. Ando ${ }^{1}$, T.C. Beers ${ }^{1}{ }^{1}$ National Astronomical Observatory, Japan; ${ }^{2}$ Michigan State University, USA.

Abstract. We have obtained high-quality spectra of 22 very metal-poor stars with Subaru/HDS, in order to conduct detailed abundance studies of the neutroncapture elements including Th. As has been found by previous abundance studies, the star-to-star scatter in the abundances of neutron-capture elements is very large. The abundance patterns of the heavy neutron-capture elements ( 56 $\leq Z \leq 70)$ in seven objects with moderate to large excesses of the neutroncapture elements are similar to that of the solar system $r$-process component. These results strongly suggest that the heavy neutron-capture elements in these objects are primarily synthesized by the $r$-process. On the other hand, the abundance ratios of the light neutron-capture elements $(38 \leq Z \leq 46)$ exhibit a rather large dispersion. Our results support previous suggestions that the light neutron-capture elements are likely to have been produced in different astrophysical sites from those associated with the production of the heavier ones. The abundances of Th in a number of these stars are slightly higher than the values expected from the solar system $r$-process pattern. The $\mathrm{Th} / \mathrm{Eu}$ ratio, which has been commonly used for nuclei cosmo-chronometry, exhibits a dispersion of about 0.3 dex in our sample.

\section{Synthetic Lick Indices as a Function of Stellar Abundance Ratios} M.L. Houdashelt ${ }^{1}$, S.C. Trager ${ }^{2}$, G. Worthey ${ }^{3}{ }^{1}$ Johns Hopkins Univ., Baltimore, USA $;{ }^{2}$ Kapteyn Astronomical Inst., The Netherlands; ${ }^{3}$ Washington State Univ., USA.

Abstract. Tripicco \& Bell (1995) used synthetic stellar spectra to characterize the sensitivities of the optical Lick indices to changes in the abundances of specific elements. These results allowed Trager et al. (2000; hereafter TFWG) to estimate the chemical abundances in elliptical galaxies from the differences between the Lick indices observed in these galaxies and those predicted by the evolutionary synthesis models of Worthey (1994). For this poster, we have calculated two new evolutionary synthesis models, one of which incorporates the TFWG chemical abundances, while the other uses standard solar abundances. We compare the integrated Lick indices from these two models and find general agreement with the differences predicted by TFWG. The only Lick indices that vary by more than two times the observational errors in these models are the $\mathrm{CN}$ indices, $\mathrm{C}_{2} 4668$, the $\mathrm{Mg}$ indices, and $\mathrm{Na} \mathrm{D}$. To examine the potential of using spectral features at redder wavelengths to further refine the TFGW abundances, we have also compared the integrated models between 0.6 and $1.0 \mu \mathrm{m}$. However, we find no spectral features that vary by more than $1 \%$ between these two models in this wavelength regime.

\section{Abundance Ratios in Ba-Poor Stars}

J. Johnson ${ }^{1}, M$. Bolte ${ }^{2}{ }^{1}$ Dominion Astrophysical Observatory, Canada; ${ }^{2} \mathrm{UCO} /$ Lick Observatory, USA.
Abstract. We present abundance ratios for four stars with $[\mathrm{Fe} / \mathrm{H}]<-3.0$ and $[\mathrm{Ba} / \mathrm{Fe}]<-1.0$. These include three stars from the sample of McWilliam et al. 
(1995, A.J, 109, 2757) which only had upper limits. These observations will be used to define the lower envelope of $[\mathrm{Ba} / \mathrm{Fe}]$ abundances in the very metal-poor stars in the Galaxy. Also, the abundance ratios of the light elements will be compared to predictions of Type II SNe ejecta and to those in Ba-rich stars to see if the stars that do not make the $r$-process can be identified.

\section{Type Ia Supernova Progenitors and Elemental Abundance Ratios C. Kobayash $i^{1}{ }^{1}$ Max-Planck-Institute for Astrophysics, Garching, Germany.}

Abstract. Elemental abundance ratios are much related to the progenitor model of Type Ia supernovae (SNe Ia). We make a comparison of the lifetime distribution function derived from several models of $\mathrm{SNe} \mathrm{Ia}$, and evaluate the SN Ia models from several observational constraints. With our single-degenerate model, the SN Ia rate in the system with $[\mathrm{Fe} / \mathrm{H}] \leq-1$ is supposed to be very small. Such metallicity effects may conflict with $[\alpha / \mathrm{Fe}]$ observed in the Damped Ly $\alpha$ systems (DLAs) and dwarf spheroidal galaxies (dSphs). However, both theoretically and observationally, SNe Ia should increase $[\mathrm{Mn} / \mathrm{Fe}]$. From the abundance pattern from $\mathrm{O}$ to $\mathrm{Zn}$, we argue that low [alpha/Fe] observed in the DLAs and dSphs is caused not by SNe Ia but by low-mass supernovae (13-15 $\left.\mathrm{M}_{\odot}\right)$, because their $[\mathrm{Mn} / \mathrm{Fe}]$ is as low as in the Milky Way halo stars.

\section{The Composition at the Outer Edge of the Galaxy}

D.A. Lubowich ${ }^{1}$, G. Brammer ${ }^{2}$, H. Roberts ${ }^{3}$, T. J. Millar ${ }^{4}$, J. M. Pasachoff ${ }^{5}$, C. Henkef $^{6}, P$. Ruffle ${ }^{4}{ }^{1}$ Hofstra University, Hempstead, NY, USA; ${ }^{2}$ STScI, Baltimore, USA $;{ }^{3}$ Ohio State University, USA $;{ }^{4}$ UMIST, Manchester, UK $;{ }^{5}$ Williams College, Williamstown, USA; ${ }^{6}$ Max-Planck Institut für Radioastronomie, Bonn, Germany.

Abstract. We present $\mathrm{cm}$ and mm-wave observations of a molecular cloud at the outer edge of the Galactic disk (kinematic galactocentric distance of $28 \mathrm{kpc}$ ). We detected $\mathrm{CO},{ }^{13} \mathrm{CO},{ }^{18} \mathrm{CO}, \mathrm{CS}, \mathrm{CN}, \mathrm{SO}, \mathrm{HCN}, \mathrm{HNC}, \mathrm{C}_{2} \mathrm{H}, \mathrm{HCO}^{+}, \mathrm{H}^{13} \mathrm{CO}^{+}$, $\mathrm{HCS}^{+}, \mathrm{NH}_{3}, \mathrm{H}_{2} \mathrm{CO}, \mathrm{C}_{3} \mathrm{H}_{2}$ and $\mathrm{CH}_{3} \mathrm{OH}$, while ${ }^{17} \mathrm{CO},{ }^{34} \mathrm{CS}$, SiO, SiS, $\mathrm{N}_{2} \mathrm{H}^{+}$, $\mathrm{DCN}, \mathrm{DNC}, \mathrm{DCO}^{+}, \mathrm{SO}_{2}$ and $\mathrm{HC}_{3} \mathrm{~N}$ were not detected. From the $\mathrm{NH}_{3}, \mathrm{H}_{2} \mathrm{CO}$, and CS data we derived a kinetic temperature of $T_{\text {kin }} \sim 20 \mathrm{~K}$ and a density of $n\left(\mathrm{H}_{2}\right) \sim 5 \times 10^{3} \mathrm{~cm}^{-3}$. The lines from $\mathrm{N}$-containing molecules that we detected were weak and we did not detect the usually strong $\mathrm{N}_{2} \mathrm{H}^{+}$or $\mathrm{HC}_{3} \mathrm{~N}$ lines. Using our 5300 chemical reaction network we calculated that this cloud is depleted in $\mathrm{N}$ by $\sim 24 \times$ and metallicity is reduced by $5 \times$ (similar to dwarf irregular galaxies or damped Lyman alpha systems) relative to the solar neighborhood. This unique composition probably results from the infall of halo gas enriched in $\mathrm{O}, \mathrm{C}$, and $\mathrm{S}$ from a burst of massive star formation shortly after the Galaxy formed. This activity would have produced both $\mathrm{O}$ and $\mathrm{S}$ which are produced by massive stars; $\mathrm{C}$ which is produced by massive and intermediate mass stars; but less $\mathrm{N}$ abundance because the secondary element $\mathrm{N}$ is produced primarily from low mass stars. Thus the edge cloud probably results from infalling halo gas that was not significantly processed during the last $10 \mathrm{Gyr}$. 


\section{The mystery of $\mathrm{CH}$ stars frequency at low metallicity}

S. Lucatello ${ }^{1}$, R.G. Gratton ${ }^{1}$, E. Carretta ${ }^{1}$, T.C. Beers ${ }^{2}$, N. Christlieb ${ }^{3}$, J.G. Cohen ${ }^{4}$ INAF, Osservatorio Astronomico di Padova, Italy; ${ }^{2}$ Michigan State University, USA; ${ }^{3}$ Hamburger Sternwarte, Germany; ${ }^{4}$ Palomar Observatory, USA.

Abstract. One of the results of the spectroscopic HK and the Hamburg/ESO surveys of metal-poor stars is the high frequency of C-enhanced stars among very metal poor stars. This is still unexplained, as well as the mechanisms responsible for the production of $\mathrm{C}$ in the few C-enhanced extremely metal poor (CEMP) stars studied so far with high resolution, high $\mathrm{S} / \mathrm{N}$ spectroscopy. The results of the follow-up works to date seem to suggest that there are different kinds of CEMP stars, exhibiting, besides the C-enhancement, $s$ - and $r$-process element enhancements, as well as normal n-capture elements abundances; and hence possibly as many $\mathrm{C}$ production mechanisms. To shed light on such mechanisms, a wider sample of CEMP stars is crucial. We present the preliminary results of abundance analysis of UVES and HIRES spectra of a sample of 10 CEMP stars, suggesting that there is no definite trend of $[\mathrm{Pb} / \mathrm{Ba}]$ with $[\mathrm{Fe} / \mathrm{H}]$, contrary to what was supposed on the basis of the Aoki et al. (2002, ApJ, 567, 1166) sample, and also at odds with the the predictions of the shell nucleosynthesis models. Moreover, the coupling of our results with those published in the literature show a clear correlation between $[\mathrm{Pb} / \mathrm{Ba}]$ and $[\mathrm{N} / \mathrm{Fe}]$, especially when considering the more metal poor stars $([\mathrm{Fe} / \mathrm{H}]<-2$ dex $)$, suggesting that the most extreme $s$-process signatures are present in stars with high $\mathrm{N}$ abundances. This is somewhat surprising, as $\mathrm{N}$ is a poison for the $s$-process and a high content of such element is expected to inhibit the $s$-process.

\section{Sulphur and Zinc Abundances in Halo and Disk stars}

P.E. Nissen ${ }^{1}$, Y.Q. Chen ${ }^{2}, M$. Asplund ${ }^{3}$, M. Pettini ${ }^{4}{ }^{1}$ University of Aarhus, Denmark; ${ }^{2}$ National Astronomical Observatories, Beijing, P.R. China; ${ }^{3} \mathrm{MSSSO}$, Australia; ${ }^{4}$ Institute of Astronomy, Cambridge, UK.

Abstract. High resolution spectra of 34 halo population dwarf and subgiant stars have been obtained with VLT/UVES and used to derive sulphur abundances from the $\lambda \lambda 8694.0,8694.6$ and $\lambda \lambda 9212.9,9237.5 \mathrm{~S}$ I lines. In addition, iron abundances have been determined from 19 Fe II lines and zinc abundances from the $\lambda \lambda 4722.2,4810.5 \mathrm{Zn}$ I lines. The abundances are based on a classical $1 \mathrm{D}$, LTE model atmosphere analysis, but effects of 3D hydrodynamical modeling on the $[\mathrm{S} / \mathrm{Fe}],[\mathrm{Zn} / \mathrm{Fe}]$ and $[\mathrm{S} / \mathrm{Zn}]$ ratios are shown to be small. We find that most halo stars with metallicities in the range $-3.2<[\mathrm{Fe} / \mathrm{H}]<-0.8$ have a near-constant $[\mathrm{S} / \mathrm{Fe}] \simeq+0.3$; a least square fit to $[\mathrm{S} / \mathrm{Fe}]$ vs. $[\mathrm{Fe} / \mathrm{H}]$ shows a slope of only $-0.04 \pm 0.01$. Among halo stars with $-1.2<[\mathrm{Fe} / \mathrm{H}]<-0.8$ the majority have $[\mathrm{S} / \mathrm{Fe}] \simeq+0.3$, but two stars (previously shown to have low $\alpha / \mathrm{Fe}$ ratios) have $[\mathrm{S} / \mathrm{Fe}] \simeq 0.0$. For disk stars with $[\mathrm{Fe} / \mathrm{H}]>-1,[\mathrm{~S} / \mathrm{Fe}]$ decreases with increasing $[\mathrm{Fe} / \mathrm{H}]$. Hence, sulphur behaves like other typical $\alpha$-capture elements, $\mathrm{Mg}$, Si and $\mathrm{Ca}$. Zinc, on the other hand, traces iron over three orders of magnitude in $[\mathrm{Fe} / \mathrm{H}]$, although there is some evidence for a small systematic Zn overabundance $([\mathrm{Zn} / \mathrm{Fe}] \simeq+0.1$ ) among metal-poor disk stars and for halo stars with $[\mathrm{Fe} / \mathrm{H}]<-2.0$. (See Nissen et al. 2003, A\&A, 415, 993) 


\section{Metallicities and Ages in the Galactic Disk}

B. Nordström ${ }^{1,2}$, J. Andersen ${ }^{1}, J$. Holmberg ${ }^{1}$, B. Jørgensen ${ }^{2}{ }^{1}$ Niels Bohr Institute, Copenhagen, Denmark; ${ }^{2}$ Lund Observatory, Sweden.

Abstract. Metallicities, ages, and full Galactic orbits have been derived for a complete, kinematically unbiased sample of $14.000 \mathrm{~F}$ and $\mathrm{G}$ dwarfs in the Solar neighborhood. The observational data are Strömgren uvby photometry, Hipparcos/TYCHO astrometry, and extensive new radial-velocity observations from which duplicity information is available for all the stars as well. The sample is magnitude complete to $V \sim 8.5$ and volume complete to $40 \mathrm{pc}$. Special attention has been given to developing and testing a new algorithm for age determination of field stars from theoretical isochrones, with particular emphasis on deriving realistic error estimates also for the oldest stars, and on identifying the stars for which no meaningful ages can in fact be derived. The results challenge traditional views of the chemical enrichment history of the Galactic disk. Full details are given in Nordström et al. (2004, A\&A, 418, 989).

\section{Sub-Damped Ly $\alpha$ Systems: Implications for the Cosmological Evolu- tion of Metals \\ C. Péroux ${ }^{1}$, M. Dessauges-Zavadsky ${ }^{2}$, S. D'Odorico ${ }^{2}$, T. Kim ${ }^{2,3}$, \\ R.G. McMahon ${ }^{3}{ }^{1}$ Osservatorio di Trieste, Italy; ${ }^{2}$ European Southern Observa- tory, Germany; ${ }^{3}$ Institute of Astronomy, Cambridge, UK}

Abstract. Damped Ly $\alpha$ Systems (DLAs), with $N(\mathrm{H} \mathrm{I})>2 \times 10^{20} \mathrm{~cm}^{-2}$, observed in the spectra of quasars have allowed us to quantify the chemical content of the Universe over cosmological scales. Such studies can be extended to lower column densities, in the sub-DLA range $\left(10^{19}<N\left(\mathrm{H}_{\mathrm{I}}\right)<2 \times 10^{20} \mathrm{~cm}^{-2}\right)$, which are systems believed to contain a large fraction of the neutral hydrogen at $z>3.5$. We use a homogeneous sample of sub-DLAs from the ESO UVES archives presented in Dessauges-Zavadsky et al. (2003, MNRAS, 345, 447), to analyze their chemical content in conjunction with a compilation of abundances from 72 DLAs taken from the literature. In particular, we analyze the $\mathrm{HI}$ column density-weighted mean abundance which is believed to be an indicator of the Universe's metallicity. The results suggest a slightly stronger evolution of this quantity in the sub-DLA range. Therefore these systems might be associated with a different class of objects which better trace the overall chemical evolution of the Universe. Finally, the elemental ratios in sub-DLAs are similar with those from DLAs. The metallicities are compared with two different sets of models of galaxy evolution in order to provide constraints on the morphology of quasar absorbers. Further details of this study can be found in Péroux et al. (2003, MNRAS, 345, 480).

\section{Sgr dSph: a Bridge between Dwarf Galaxies and DLAs?}

L. Sbordone ${ }^{1}, 2$, P. Bonifacio ${ }^{3}$, G. Marconi ${ }^{1}$, L. Pasquini ${ }^{4}$, V. Hill ${ }^{5}{ }^{1}$ European Southern Observatory, Santiago, Chile; ${ }^{2}$ Dipartimento di Fisica, Universita di Roma, Italy; ${ }^{3}$ INAF-OA, Trieste, Italy; ${ }^{4}$ European Southern Observatory, Germany; ${ }^{5}$ Observatoire de Paris-Meudon, France.

Abstract. We present abundances for 12 giants in the Sagittarius Dwarf Spheroidal Galaxy (Sgr dSph) obtained from VLT-UVES spectra. Moving on a 
short period, polar orbit around the Milky Way, the Sgr dSph is undergoing tidal disruption and will eventually dissolve in the Galactic Halo. Our sample appears dominated by a Fe-rich, $\alpha$-element-poor and young population, indicative of a long chemical processing of the $\mathrm{dSph}$ gas during a slow, probably bursting star formation history. This population is the most metal-rich ever observed in a dwarf galaxy of the local group, and has the lowest $\alpha$-element content. The "extreme" composition observed in the Sgr dSph may be attributed to an unusually prolonged star formation, related to its strong interaction with the MW. Placing the known abundances of the $\mathrm{LG}$ dwarfs on the $[\alpha / \mathrm{Fe}]$ vs. $[\mathrm{Fe} / \mathrm{H}]$ plane allows now to recognize a well defined evolution sequence. The "dwarf sequence" resembles the one followed by the MW disc star, but at a consistently lower $[\alpha / \mathrm{Fe}]$ ratio for each given $[\mathrm{Fe} / \mathrm{H}]$. Conversely, it is apparently superimposed to the one followed by many of the Damped Lyman alpha systems.

\section{Bimodal Metallicity Distribution Function of OLD Stars}

T. Shigeyama ${ }^{1}, T$. Tsujimoto ${ }^{1}, Y$. Yoshii ${ }^{1}{ }^{1}$ National Astronomical Observatory, University of Tokyo, Japan.

Abstract. The external pollution of the first stars in the Galaxy is investigated. The first stars were born in clouds composed of the pristine gas without heavy elements. These stars accreted gas polluted with heavy elements while they still remained in the cloud. As a result, it is found that they exhibit a distribution with respect to the surface metallicity that is easily distinguished from the metallicity distribution of Population II stars. This metallicity distribution function strongly suggests that the recently discovered most metal-deficient star HE0107-5240 with $[\mathrm{Fe} / \mathrm{H}]=-5.3$ was born as a metal-free star and accreted gas polluted with heavy elements. Future observations for a number of metaldeficient stars with $[\mathrm{Fe} / \mathrm{H}]<-5$ will be able to prove or disprove this external pollution scenario.

\section{CS 29497-030: Lead in the Early Galaxy}

T. Sivarani ${ }^{1}$, P. Bonifacio ${ }^{1}$, P. Molaro ${ }^{1}$, R. Cayrel $^{2}$, M. Spite ${ }^{2}, F$. Spite $^{2}$, B. Plez $^{3}$, J. Andersen ${ }^{4}$, B. Barbuy ${ }^{5}$, T. C. Beers ${ }^{6}$, E. Depagne ${ }^{7}$, V. Hill ${ }^{2}$, P. François $^{2}$, B. Nordström ${ }^{4,8}$, F. Primas ${ }^{7}{ }^{1}$ INAF-OA, Trieste; ${ }^{2}$ Observatoire de Paris-Meudon; ${ }^{3}$ GRAAL-Université de Montpellier; ${ }^{4}$ Astronomical Observatory, Copenhagen; ${ }^{5}$ IAG, Universidade de São Paulo; ${ }^{6}$ Michigan State University; ${ }^{7}$ European Southern Observatory; ${ }^{8}$ Lund Observatory, Sweden.

Abstract. We present an abundance analysis of the halo blue straggler CS 29497030, based on high-resolution, high S/N spectra from the ESO VLT/UVES. The star has very low metallicity $([\mathrm{Fe} / \mathrm{H}]=-2.8)$, large overabundances of $\mathrm{C}$, $\mathrm{N}$, and $\mathrm{O}([\mathrm{C} / \mathrm{Fe}]=+2.38,[\mathrm{~N} / \mathrm{Fe}]=+1.88$, and $[\mathrm{O} / \mathrm{Fe}]=+1.67)$, and large enhancements of heavy $s$-process elements. Most strikingly, the $\mathrm{Pb}$ enhancement $([\mathrm{Pb} / \mathrm{Fe}]=+3.5)$ is the highest yet observed in very metal-poor $s$-process rich stars. The occurrence of several very metal-poor stars with large overabundances of heavy $s$-process elements, e.g $\mathrm{Ba}, \mathrm{La}, \mathrm{Ce}, \mathrm{Nd}$, and particularly $\mathrm{Pb}$, suggests that the $s$-process was operating at early times in the Galaxy, at least locally. This appears to contradict the long-accepted view that the neutron capture elements in low-metallicity stars originate from the $r$-process only. However, 
like some (but not all) similar stars, CS 29497-030 is a binary ( $\mathrm{P}=342 \mathrm{~d}$ ), and the $\mathrm{Pb}$ and other $s$-process elements could have been synthesized in the envelope of a former AGB companion, perhaps significantly later than the formation of the star itself. Full details of our analysis and discussion are reported in Sivarani et al. (A\&A accepted).

\section{Abundance of Nitrogen in the Early Galaxy from the NH Band at $336 \mathrm{~nm}$}

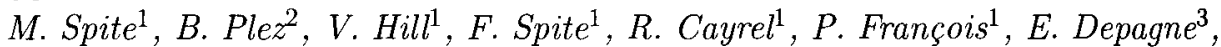
J. Andersen ${ }^{4}$, B. Barbuy ${ }^{5}$, T. Beers ${ }^{6}$, P. Bonifacio ${ }^{7}$, P. Molaro ${ }^{7}$, B. Nordström ${ }^{4,8}, F$. Primas ${ }^{3}{ }^{1}$ Observatoire de Paris-Meudon; ${ }^{2}$ GRAAL-Université de Montpellier; ${ }^{3}$ European Southern Observatory; ${ }^{4}$ Astronomical Observatory, Copenhagen; ${ }^{5}$ Universidade de São Paulo; ${ }^{6}$ Michigan State University; ${ }^{7}$ INAFOA Trieste; ${ }^{8}$ Lund Observatory, Sweden.

Abstract. As part of the ESO Large Programme "First Stars", high-resolution, high-S/N spectra of 35 extremely metal-poor giants selected from the HK survey (Beers et al. 1992, AJ, 103, 1987; 1999, AJ, 117, 981) have been obtained at the VLT. The spectra were analyzed with the LTE spectral line code "Turbospectrum" and OSMARCS model atmospheres (Gustafsson et al. 1975, Plez et al. 1992, Asplund et al. 1997). Element abundances from $\mathrm{C}$ to $\mathrm{Zn}$ are presented in Cayrel et al. (2003, A\&A, 416, 117), but nitrogen abundances from the CN band are lacking for most of the stars. We have used the $\mathrm{NH}$ band at $336 \mathrm{~nm}$ to determine nitrogen abundances for all our stars. The dispersion in the relations of $[\mathrm{N} / \mathrm{Fe}]$ vs. $[\mathrm{Fe} / \mathrm{H}]$ (and $[\mathrm{C} / \mathrm{Fe}]$ vs. $[\mathrm{Fe} / \mathrm{H}]$ ) is very large, However, a group of stars displays very low values of $\mathrm{C} / \mathrm{N}$, suggesting that their atmospheres are mixed with internal layers where the $\mathrm{CN}$ cycle has converted $\mathrm{C}$ to N. Supporting this theory, the relation $(\mathrm{C}+\mathrm{N})$ vs. $[\mathrm{Fe} / \mathrm{H}]$ is flat, and the dispersion around the mean value is much smaller. For unmixed stars with $[\mathrm{Fe} / \mathrm{H}]<-3.4,[\mathrm{~N} / \mathrm{Fe}]$ is close to +0.1 and $[\mathrm{N} / \mathrm{O}]$ close to -0.6 , suggesting these ratios for the primordial production of $\mathrm{N}$.

\section{Behavior of Sulfur Abundances in Halo Stars Observed with HIDES at OAO}

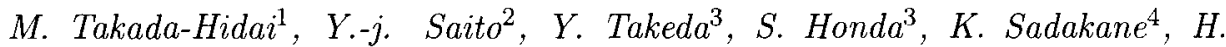
Izumiura ${ }^{5}$, S. Masuda ${ }^{5}{ }^{1}$ Liberal Arts Education Center, Tokai University, Japan; ${ }^{2}$ Physics Department, Tokai University, Japan; ${ }^{3}$ National Astronomical Observatory of Japan, Tokyo; ${ }^{4}$ Osaka Kyoiku University, Kashihara, Japan; ${ }^{5}$ National Astronomical Observatory of Japan, Okayama .

Abstract. LTE and non-LTE (NLTE) abundances of sulfur of 21 metal-poor stars (mainly halo stars) and one normal star were explored in the metallicity range of

$-3<[\mathrm{Fe} / \mathrm{H}]<0$, using high-resolution $(\sim 50000)$, high-signal-to-noise $(\sim 100$ 450) spectra of $\mathrm{S}$ I lines with the multiplet numbers of 1 and 6 , which were observed with the $1.88 \mathrm{~m}$ telescope equipped with the High Dispersion Echelle Spectrograph (HIDES) at the Okayama Astrophysical Observatory (OAO). Equivalent widths of $\mathrm{S}_{\mathrm{I}}(1)(9212,9228,9237 \AA)$ and $\mathrm{S}_{\mathrm{I}}(6)(8693.9,8694.6 \AA)$ lines were analyzed to obtain the abundances. Iron abundances were determined from 
Fe II lines and used to investigate the behavior of $\mathrm{S}$ against Fe. Our main results are: (1) The LTE abundances of SI(6) are systematically smaller than those of $\mathrm{SI}(1)$ with the average difference of $0.07 \pm 0.09 \mathrm{dex}$. (2) The NLTE corrections for $\mathrm{SI}_{\mathrm{I}}(6)$ abundances are in the range from -0.01 to -0.05 dex with the average of -0.03 dex, while those for $\mathrm{SI}(1)$ are from -0.14 to -0.33 dex with the average of -0.22 dex. Consequently, NLTE abundances of SI(6) are systematically larger than $\mathrm{SI}(1)$ with the average of $0.13 \mathrm{dex} \pm 0.14$ dex. (3) As for the behavior of NLTE $[\mathrm{S} / \mathrm{Fe}]$, it forms a plateau with the average of $[\mathrm{S} / \mathrm{Fe}]=+0.49 \pm 0.12 \mathrm{dex}$ in the range of $-3<[\mathrm{Fe} / \mathrm{H}] \leq-1$.

\section{Chemical Evolution of MgII Absorption Systems}

T. Tsujimoto ${ }^{1}, N$. Kobayashi ${ }^{1}, M$. Iy $e^{1}, Y$. Yoshii ${ }^{1}{ }^{1}$ National Astronomical Observatory, University of Tokyo, Japan.

Abstract. Based on the $[\mathrm{Mg} / \mathrm{Fe}]$ ratios for the high- $\mathrm{Mg}$ II absorption systems obtained by the near-infrared camera and spectrograph for the Subaru $8.2 \mathrm{~m}$ Telescope, we construct the chemical evolution model for these high-z objects. It is found that host galaxies for these systems formed within a short time scale of a few hundred million years, suggesting that they are progenitors of modernday dwarf galaxies. The elemental abundance relation between stars and gas in dwarf galaxies will also be discussed.

\section{The Heavy Element Abundance Pattern in Lead Stars}

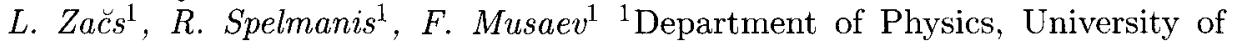
Latvia.

Abstract. A quantitative understanding of evolution of nuclei heavier than iron in the Galaxy has so far been a challenging problem. Only recently have the observational data grown in number and precision to allow a direct comparison with theoretical predictions. In order to reconstruct the evolutionary history of neutron-capture elements in the Galaxy, one must disentangle the $s^{-}$and $r$-process contributions of all their isotopes and follow their abundances as a function of Galactic age. A new series of abundance analysis of lead star candidates is presented here starting with spectroscopy of the lead-rich star HD196944 $([\mathrm{Fe} / \mathrm{H}]=-2.45 ;[\mathrm{hs} / \mathrm{ls}]=+0.8)$. High resolution CCD spectra in a large spectral region from 3500 to $10000 \AA$ have been used to determine detailed abundance patterns for a large number of species. The results are compared with available spectroscopic observations of stars at different metallicities in both the Galactic halo and the disc and with calculations of neutron-capture nucleosynthesis. 